九州大学学術情報リポジトリ

Kyushu University Institutional Repository

\title{
Structural features of polyacrylonitrile-based carbon fibers
}

Li, Wei

Institute for Materials Chemistry and Engineering, Kyushu University

Long, Donghui

State Key Laboratory of Chemical Engineering, East China University of Science and Technology

Miyawaki, Jin

Institute for Materials Chemistry and Engineering, Kyushu University

Qiao, Wenming

State Key Laboratory of Chemical Engineering, East China University of Science and Technology

他

http://hdl. hand le. net/2324/27284

出版情報：Journal of Materials Science. 47 (2)，pp.919-928，2012-01-01. Springer バージョン：

権利関係: (C) Springer Science+Business Media, LLC 2011 


\section{Structural features of polyacrylonitrile-based carbon}

\section{fibers}

Wei $\mathrm{Li}^{\dagger}$, Donghui Long ${ }^{\ddagger}$, Jin Miyawaki ${ }^{\dagger}$,Wenming Qiao ${ }^{\ddagger}$, Licheng Ling ${ }^{\ddagger}$, Isao Mochida ${ }^{\dagger}$, Seong-Ho

$$
\text { Yoon }^{*}+
$$

† Institute for Materials Chemistry and Engineering, Kyushu University, Kasuga, Fukuoka 816-8580, Japan

‡ State Key Laboratory of Chemical Engineering, East China University of Science and Technology, Shanghai 200237

\footnotetext{
* Corresponding author: Seong-Ho Yoon, Tel: +81 92583 7959, Fax: +81 925837897.

E-mail: yoon@cm.kyushu-u.ac.jp
} 


\begin{abstract}
The structural changes as functions of spinning conditions and heat treatments were investigated with respect to the structural feature of PAN-based carbon fibers by scanning tunnelling microscopy (STM). The distinct granule structure on the cross section of both high tensile strength (HS) and high modulus (HM) carbon fibers was observed by SEM, while slender granule-shape domain on the longitudinal surface was revealed by STM. A structure model was proposed, which depicted that the PAN-based carbon fiber was a heterogeneous structure composed of aggregated mesostructural domains. These domains were closely arranged into spiral form along fiber axis, allowing the fibers have high strength and good elongation. The initial shape and size of domains was determined by the precursor composition and spinning conditions and also strongly depended on the heat-treated temperature and stretching conditions. The smaller or slender domain, the higher tensile strength obtained for fibers. We expect that the PAN-based carbon fiber with better performance should be produced by optimizing the size and shape of these domains.
\end{abstract}

Keyword: Microstructure, PAN based carbon fiber, mechanical properties 


\section{Introduction}

Carbon fiber is a strategic engineering material which is greatly successful to be commercialized over tens of decades. Its high strength and modulus, combined with the light weight and superior chemical and thermal stability are making it much more attractive for high-volume applications ranging from high-performance sporting goods to space and aircraft structures for energy-saving through the lightweightening [1-6]. Carbon fibers can be produced from various precursors, but generally PAN, rayon and pitch are used for large-scale production [7-9]. Among them, PAN is most preferred because the resulting carbon fibers have relatively high strength, elongation and the lowest volume fraction of voids [10]. PAN-based carbon fibers are produced by the oxidative stabilization of PAN precursor fibers [11], normally followed by a two-stage carbonization process, with an added heat treatment stage to manufacture a high modulus fiber $[12,13]$. The final mechanical properties are, to a great extent, determined by the precursors, the processes and the conditions used to fiber production [14-17].

Continuous researches have now realized PAN-based carbon fibers with tensile strengths over of 7.0 GPa (Toray T-1000G). The most featuring aspect of such a fiber is its higher ratio of strain to failure of $2.4 \%$ with comparing to that of $1.5 \%$ which could be normally achieved in ordinary PAN-based carbon fibers. Also a great progress has been made to improve the modulus of PAN-based carbon fibers. It has, for example, gone up from 230 GPa (Toray T300) in early 1980 to 560 GPa (Toray M60). These improvements have been possible partly through better controlling of processing conditions and specifically, in authors' opinion, due to the optimization of precursor composition and spinning method.

The structure and properties of materials are closely related, which meets with almost universal acknowledgement. It follows that to understand the properties of a material, information on structure is vitally important. In the pursuit of ever increasing performance, the structure of carbon fibers, and the differences in structure produced by various processing routes, has been the subjects of close scrutiny. However, comparing the flourishing commercial applications, the study of PAN-based carbon fibers from scientific view, especially in the relation of microstructure and properties, is relatively lagging and in downturn. There is indeed a vast amount of earlier literature on the structural analysis of carbonized 
and graphitized PAN-based fibers [8-26]. Most of them have been based on the information from the $\mathrm{XRD}$ and TEM results, the former giving a macroscopic mean measurement of dimensions, and the latter permitting the local orientation of individual units to be scrutinized but only in two dimension, neither of which gives a direct three-dimensional observation of structure. Several structural models of PAN-based carbon fiber have been proposed, such as a ribbon model [27], a basket-weave model [2], a core-skin model [28] and a crumpled sheet model [29, 30]. Among these, the crumpled sheet model proposed by Guigon et al. was generally accepted, which provided a delicate impression of high strength (HS) and high modulus (HM) PAN-based carbon fibers. It was concluded that the HS carbon fibers were composed of two-dimension turbostratic graphitic crystallites poorly oriented preferentially parallel to the fiber axis and have a two-phase structure ( crystalline and amorphous phases ). And the HM carbon fibers were made up of slowly undulating graphite ribbons highly oriented along the fiber axis. Unfortunately, all of these models are on isolated aspects on the two-dimensional structure and so fail to provide a comprehensive cognition of PAN-based carbon fibers in a multi-level scope. It is necessary to conduct extensive research on the morphological and microstructural aspects of carbon fibers in order to improve our understanding of structure and properties of these critical functional materials.

In the present study, high resolution SEM and STM were used to provide direct insight into understanding and modeling the mesostructure of PAN-based carbon fibers. The three-dimensional nature of SEM and STM imaging permits a clearer visualization and more detailed examination of regional structures. Based on these observations, a domain structural model from the microscopic and mesoscopic view has been proposed. The shape and size of these domains depended on the precursor structure and process condition have briefly correlated with the properties of the carbon fiber. It is our belief that such information could provide some implications for optimizing process conditions and for deliberately controlling the structure to achieve high strength and high modulus PAN-based carbon fibers.

\section{Experimental Section}




\subsection{Materials}

Samples in this study were directly supplied from company (Toray Co., Japan). Carbon fibers for this study were the PAN-based Torayca T300, T800H and M55J carbon fibers (Toray Co., Japan). They were supplied from the factory without any surface treatment and sizing. The precursor fibers for T300, T800H which were stabilized states were also supplied. The T300 precursor fibers were further heattreated at 1500,2000 and $2500{ }^{\circ} \mathrm{C}$ for 10 min under the argon atmosphere performed under the standard preparation of T300. The obtained fibers were denoted as HT-X, where X is the heat-treatment temperature. In addition, a series of PAN-based carbon fibers with the different preparation conditions, such as long-time carbonization, high elongation, and high co-polymer content were also supplied from the factory line for comparative investigations. The precise processing conditions are proprietary, only information provided was that the above-mentioned condition differed with the standard preparation process of T300.

Naphthalene-derived mesophase pitch based carbon fibers were supplied from Mitsubishi Gas Chemical Company for comparison investigations.

\subsection{Characterization}

The longitudinal and transverse cross sections of carbon fibers were examined using field emission SEM (JSM-6320F, JEOL). To examine the transverse alignments under SEM, all fiber samples were cut in liquid nitrogen to approximately $0.1 \mathrm{~cm}$ length, and attached to a copper grid with graphite paste. The transverse section exposed by the cutting was observed without any coating. The longitudinal section of carbon fibers was observed using STM (Nanoscope IIIa, Digital Instrument, USA). STM images were acquired in a constant current mode, using tunneling currents in the range of 0.2-1.0 nA, bias voltage in the range of $0.1-1 \mathrm{~V}$, and scan frequencies of $1-2 \mathrm{~Hz}$, depending on the scan size and the tunnel current applied.

\section{Results}




\subsection{Mesostructure of PAN-based Carbon Fibers}

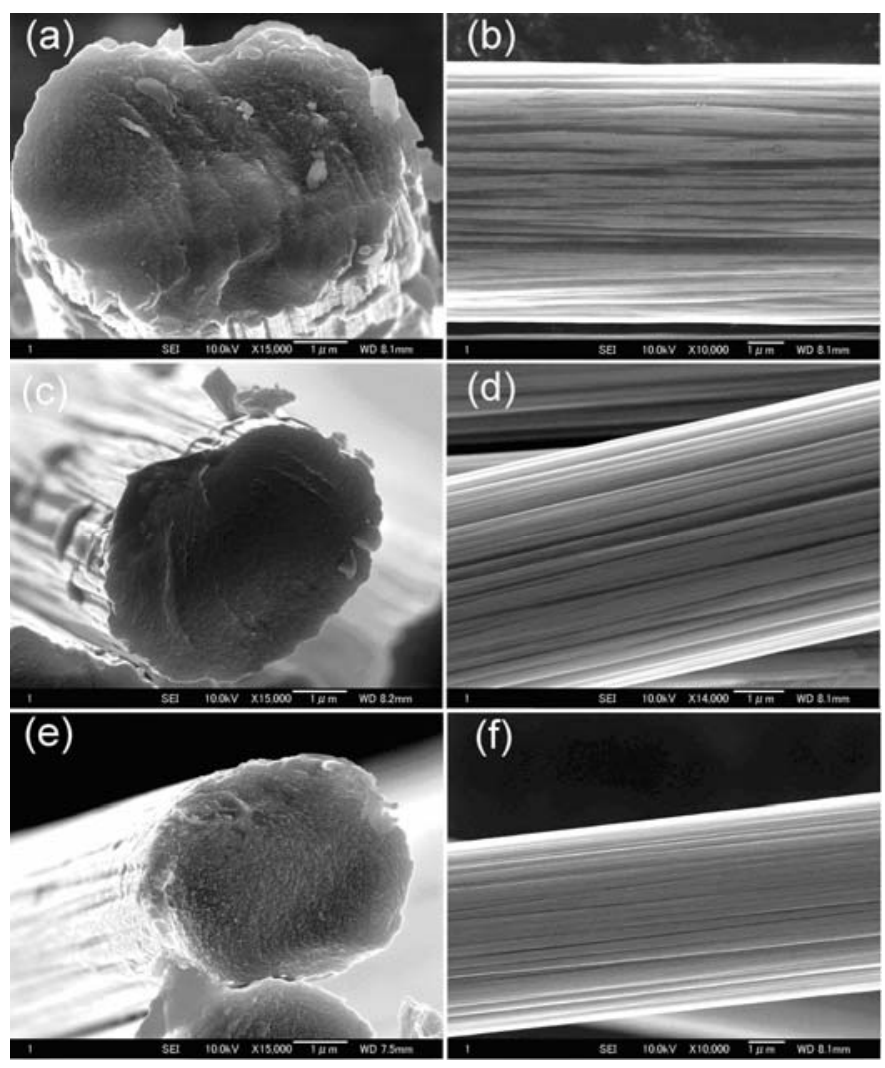

Figure 1 The transverse and longitudinal SEM images of T300 (a, b), T800H (c, d) and M55J (e, f ) carbon fibers

PAN-based carbon fibers of T300, T800H and M55J have been examined by SEM. The fibers T300 and T800H are known as HS carbon fibers while M55J fibers are regarded as HM carbon fiber. The low magnification images of the transverse and longitudinal sections of these fibers are given in Figure 1. The external morphologies of these fibers are very similar: circular cross sections and long grooves parallel to fiber axis. However, the fiber diameter and width of groove exhibit differences and are both in the order of $\mathrm{T} 300>\mathrm{T} 800 \mathrm{H}>\mathrm{M} 55 \mathrm{~J}$. It seems that the cross sections of all fibers consist of nearly isotropic surface. No radially orientation or core-sheath distinct structure is observed. In addition, the flaws or voids, which are believed to the determined strength-limiting factor [31], are also not found throughout the comprehensive SEM observation. 


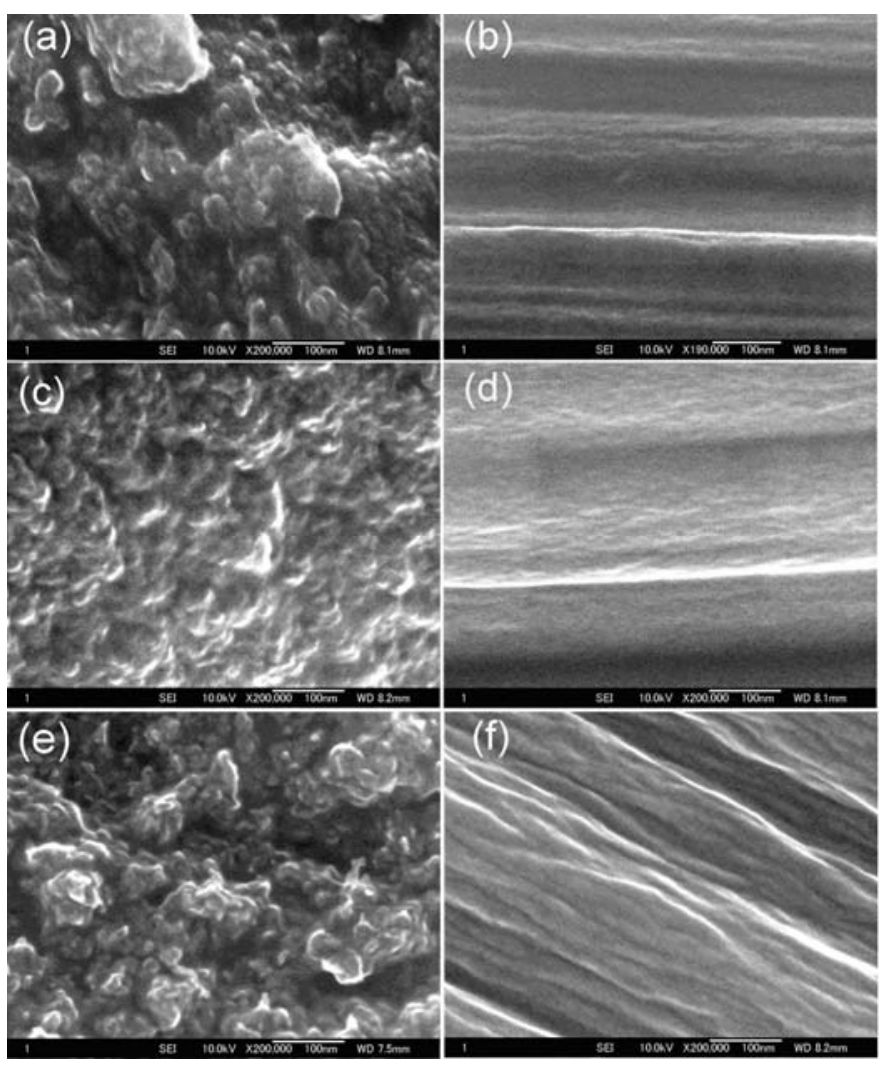

Figure 2 High-resolution transverse (left) and longitudinal (right) SEM images of T300 (a, b), T800H (c, d) and M55J (e, f) carbon fibers

The high magnification SEM images shown in Figure 2 reveal clearly that the cross section of T300 fibers consists of poorly defined aggregated granules with the size of 10-30 nm. The enlarged longitudinal sections show some wave-like stripes along the fiber axis. The T800H fibers, having higher strength, have a similar morphology with that of T300 fiber, but show a relatively small granule size and smooth axis surface. The M55J fiber shows a clearly melted granule structure in the cross sections and more striking wave feature on the skin surface. The similarities in general appearance of these fibers indicate that PAN-based carbon fibers have some common surface characters. The differences in the fine texture are probably attributable to use of different precursor compositions and processing conditions, which is reflected in the property discrepancies of these fibers. 


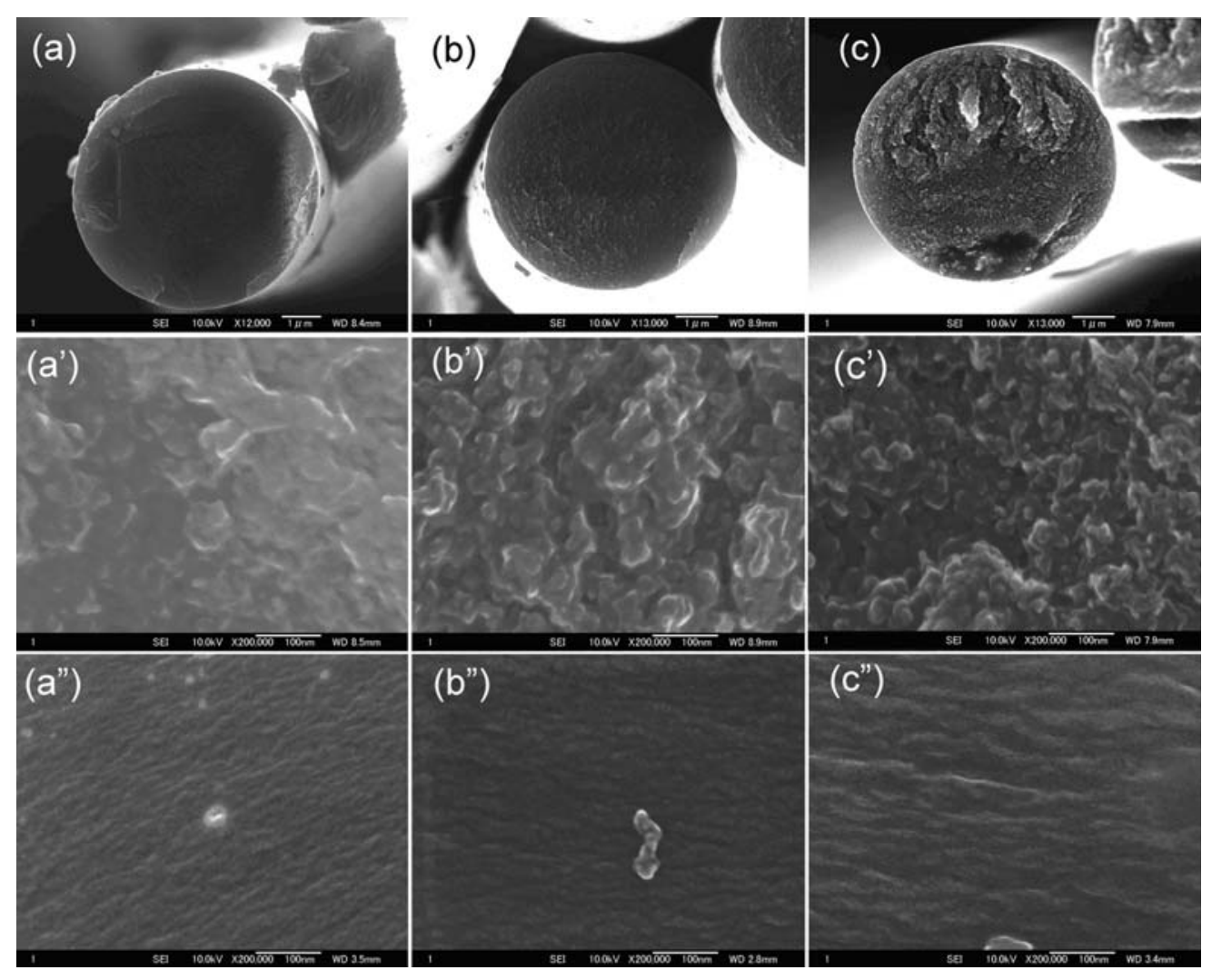

Figure 3 Transverse, enlarged transverse and longitudinal SEM images of HT-1500 (a, a', a”), HT-2000 (b, b’ b”) and HT-2500 (c, c', c”)

Figure 3 shows the changes of transverse and longitudinal structures of round shaped PAN-based carbon fibers as a function of heat treated temperature. It is known that high temperature carbonization $\left(1500^{\circ} \mathrm{C}\right)$ produce carbon fiber the HS while high temperature graphitization $\left(2500^{\circ} \mathrm{C}\right)$ produce the $\mathrm{HM}$ carbon fiber. To reveal the microstructural differences between HS and HM carbon fibers, PAN-based precursor fibers were heat-treated at 1500, 2000 and $2500{ }^{\circ} \mathrm{C}$, respectively provided by Toray Co. The granule distribution of each carbon fiber appeared very homogeneous on the transverse section regardless of heat treatment temperature, however, the image definition of granular increased with the temperature increasing. 


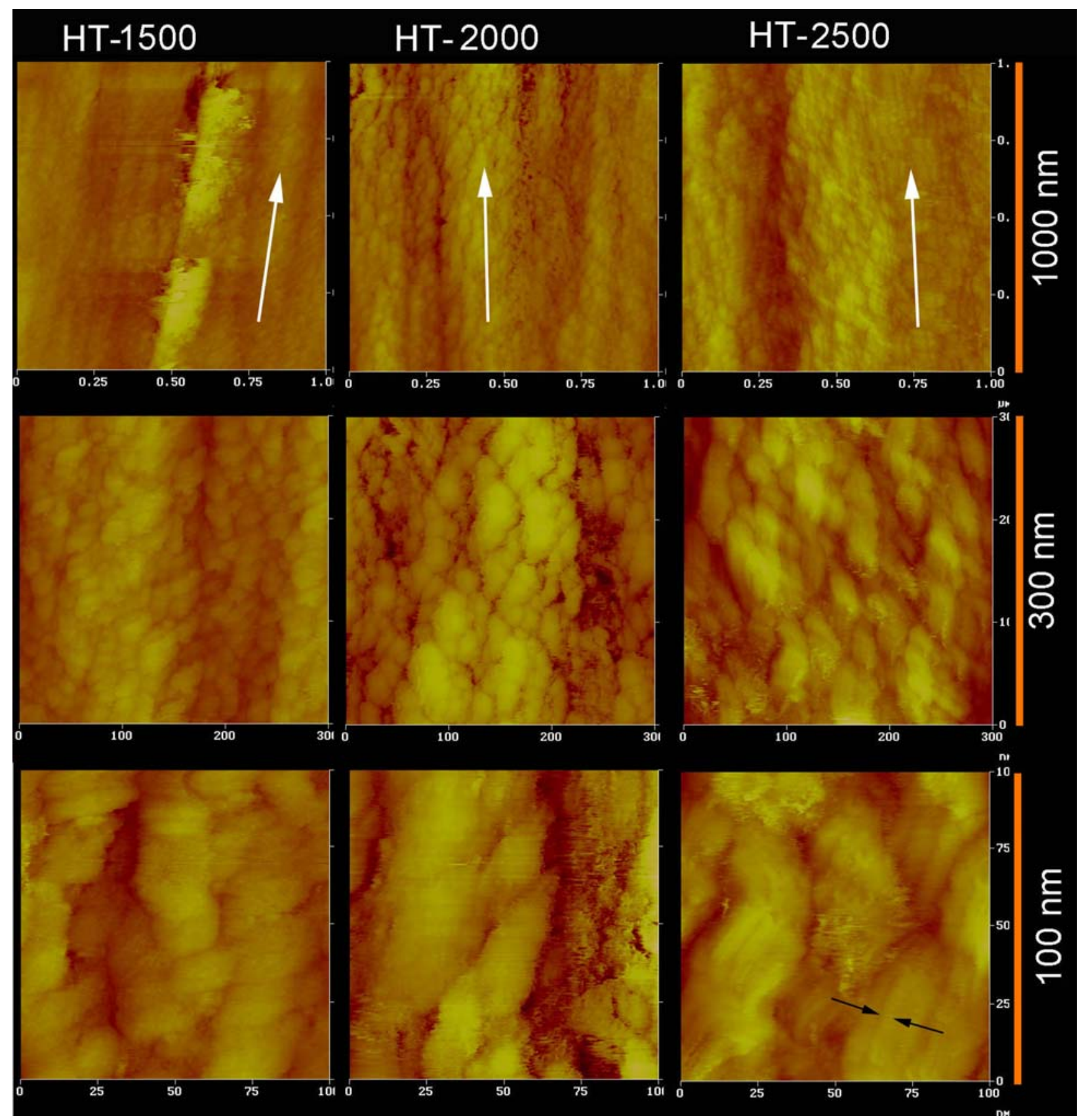

Figure 4 STM images of HT-1500, HT-2000 and HT-2500 scanned at different magnifications. (white arrow indicates the fiber axis direction)

Figure 4 presents the STM images observed on the three carbon fibers heat-treated at different temperatures scanned at various scales. For scans at $1000 \mathrm{~nm}$, in all case, the similar surface roughness is clearly revealed and the extruding striations along the fiber axis, as observed by SEM, are evident. However, it is difficult to use SEM to obtain the surface details at these scales $\left(1000 \mathrm{~nm}^{2}\right.$ corresponding 
to a magnification of ca. 30000 times). The STM images at higher magnification reveal that all fibers have a rock-stacking surface. These rocks, defined as mesodomains, connect predominately end to end or side to side, and twist frequently, forming a spiral twine-like feature oriented preferentially along the fiber axis. On each rock, the needle-shaped voids with a diameter of 2-10 nm are easily observed. The differences in the surface features among these heat-treated carbon fibers can be discerned. Two points should be underlined. First, with the increase of the heat-treated temperature, the size of rocks increases significantly. It is about $15-25 \mathrm{~nm}$ by $25-50 \mathrm{~nm}$ for the fiber HT-1500, $25-35 \mathrm{~nm}$ by $30-80 \mathrm{~nm}$ for the fiber HT-2000, and 30-40 nm by 50-80 nm for HT-2500. It should be noticed that the width of these rocks is almost similar to the diameter of granules on the cross sections observed by SEM. Secondly, the ribbon structure having diameter of ca. $7 \mathrm{~nm}$ (noted in Figure 4), which can be readily detected on the graphitized fiber HT-2500 surface in the highest magnification image, are not found on the surface of others fibers.

\subsection{STM Observation of PAN-based Carbon Fibers Prepared at Different Process Conditions}

Figure 5 displays the STM images of the external surface of carbon fibers prepared at high elongation, long-time carbonization and high co-polymer content, respectively. The tensile strength of these fibers were known as high elongation> long-time carbonization> high co-polymer content, which is provided by the manufacturer. All fibers have the similar rock-stacking feature, further confirming the existing of mesodomain in the PAN-based carbon fibers. However, the differences of tensile strength should be related to the shape and size of mesodomain. Comparing the three fibers, high elongation gives an obviously slender shape, while long-time carbonization results in intermediate size and high co-polymer content produces the largest size. 


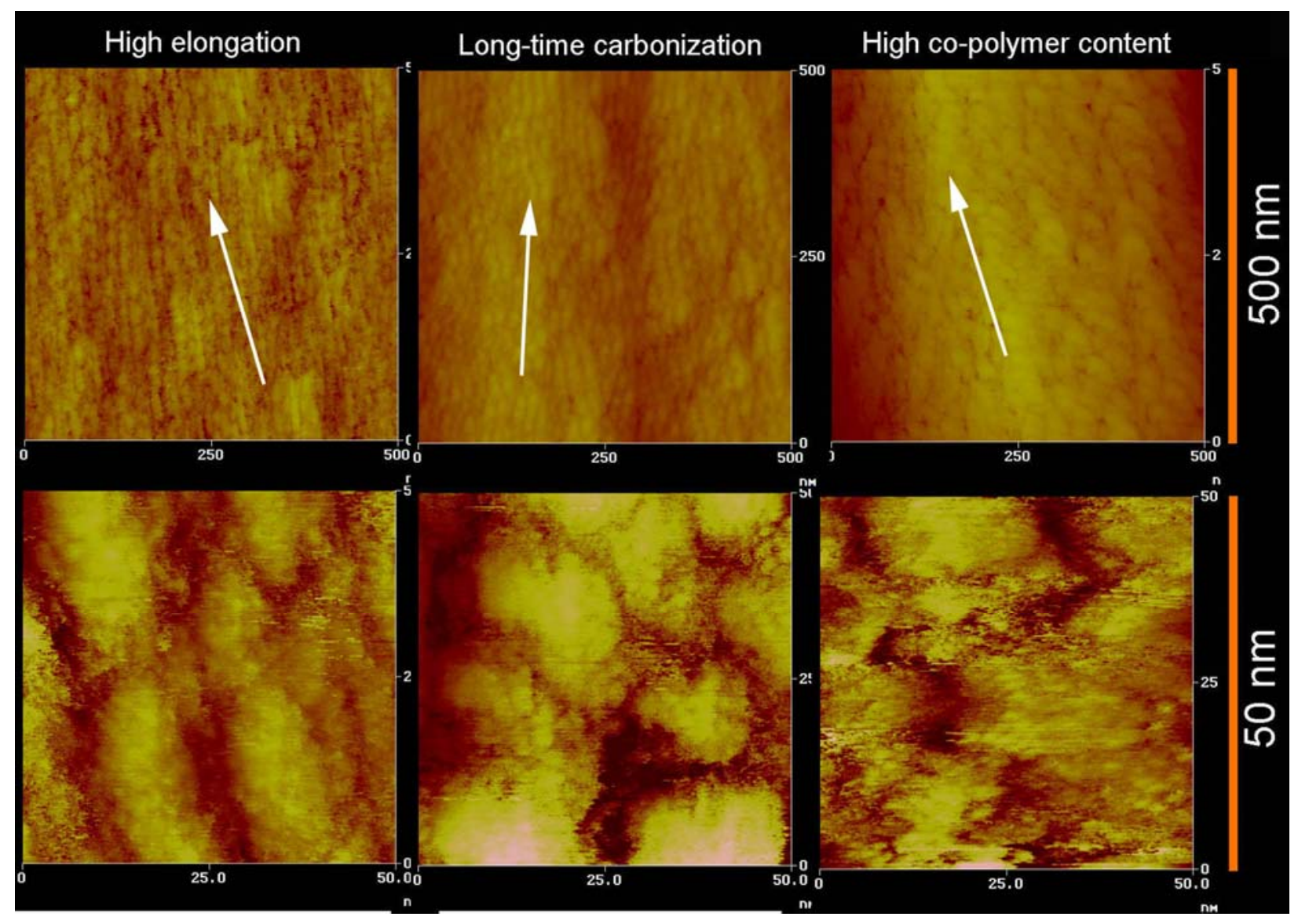

Figure 5 STM images of carbon fibers prepared at high elongation, long-time carbonization and high co-polymer content scanned at different magnifications (white arrow indicates the fiber axis )

\subsection{STM comparison of the mesostructures of PAN-based and mesophase pitch-based carbon fibers}

In our earlier work, we have extensively studied the domain structure of the mesophase pitch-based carbon fibers [8, 32-35]. As a comparison, Figure 6 gives the typical STM images for PAN-based and pitch-based carbon fibers. And Figure 7 depicts the resultant schematic model of arrangement of domains. The uniform granules having size of ca. $80 \mathrm{~nm}$ in length and ca. $70 \mathrm{~nm}$ in diameter are clearly observed in the mesophase pitch-based carbon fibers, which inherit from the organic polymeric structures of the liquid crystalline mesophase [36]. Especially, the structural feature of such domains in the mesophase pitch-based carbon fibers shows the linear and ordered arrangement to the fiber axis. In comparison, the PAN-based carbon fibers consist of irregular twisted domains arranged with spiral form, giving an impression of the spiral twine-like structure. Similar ribbon structures having diameter of ca. $7 \mathrm{~nm}$ can be clearly observed in the highest magnification images for both kinds of fibers. However, 
poor linearity of ribbon from PAN-based carbon fiber is observable than the mesophase pitch-based carbon fiber, and the shape of the former is relatively irregular.

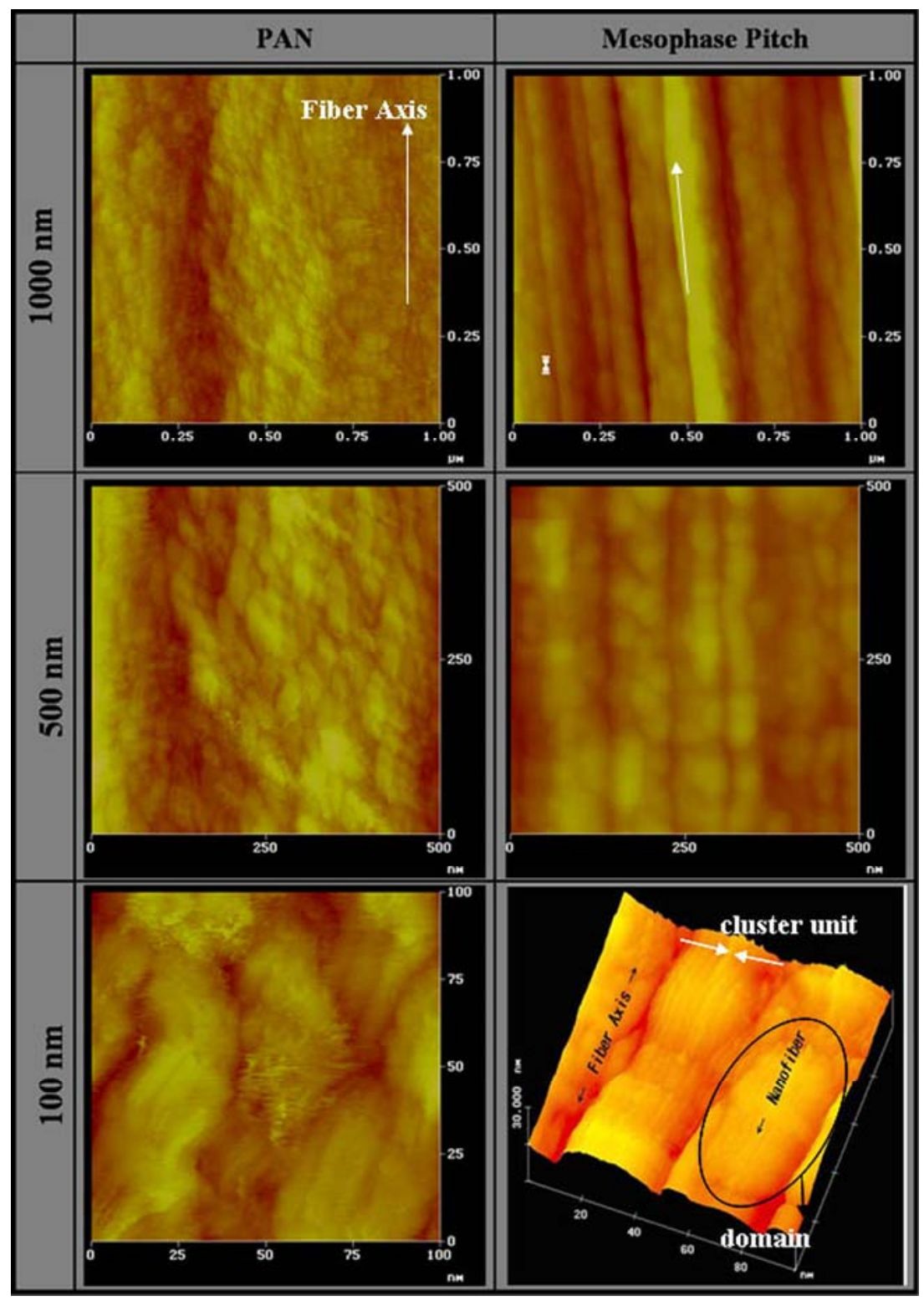

Figure 6 STM images of PAN-based and mesophase pitch-based carbon fibers 


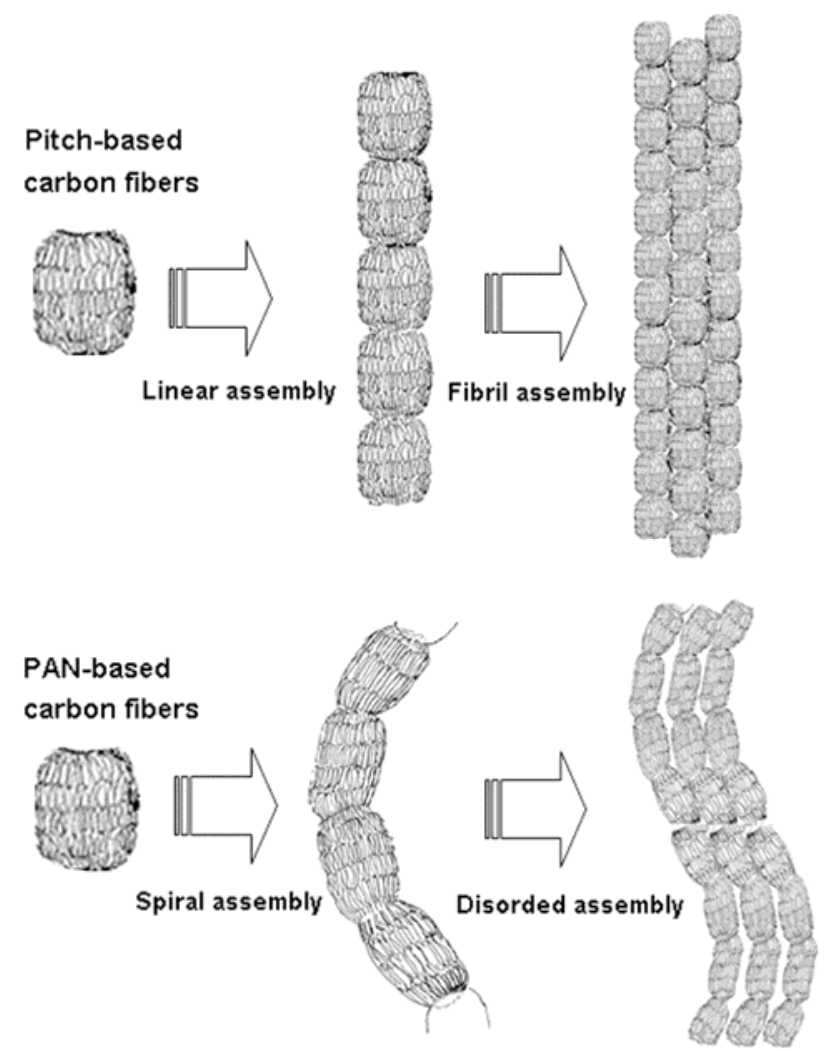

Figure 7 Schematic model of arrangement of domains in mesophase pitch-based and PAN-based carbon fibers

\section{Discussion}

\subsection{Structural model of PAN-based carbon fibers}

From the earlier two-dimension observations [29, 30], it can be concluded that the HS carbon fibers are composed of two-dimension turbostratic graphitic crystallites poorly oriented preferentially parallel to the fiber axis and have a two-phase structure ( crystalline and amorphous phases ). And the HM carbon fibers can described as made up of ordered graphite ribbons highly oriented along the fiber axis. We also observed the similar results using TEM, which are shown in the Supporting Information. However, these existing models cannot explain the distinctly granule texture on the cross-section observed by SEM and rock-stacked feature on the longitudinal section revealed by STM. It seems that there is a lost bridge between the carbon crystallite and the bulk fiber. From our experimental observations and the various pieces of evidence gathered from literature [35-38], a new model based on 
the mesostructured domain is proposed for PAN-based carbon fiber. The schematic representation of this model was depicted in Figure 8. The PAN-based carbon fiber has been divided into two different structural levels including microscopic and mesoscopic textures. At the microscopic level, carbon fiber was composed of carbon microcrystallines. The microcrystalline incorporates such features as packing, orientation, interweaving, twisting, folding, imperfect stacking, dislocation and microvoids et al, which have been widely revealed by TEM observation in the earlier references [25-29]. Mesodomain is the next level, which are constituted by closely packed microcrystallines. The mesodomain has an irregularly quasi-cylindrical shape with the size of $20-40 \mathrm{~nm}$ in diameter and $30-80 \mathrm{~nm}$ in length as revealed by STM and SEM. These mesodomains connect closely either end to end or side to side each other, twist and entangle continually, disorderly align to an aggregated fiber texture along the fiber axis. That is to say, the PAN-based carbon fiber was a heterogeneous system of aggregated mesodomains with preferred orientation parallel to the fiber axis.

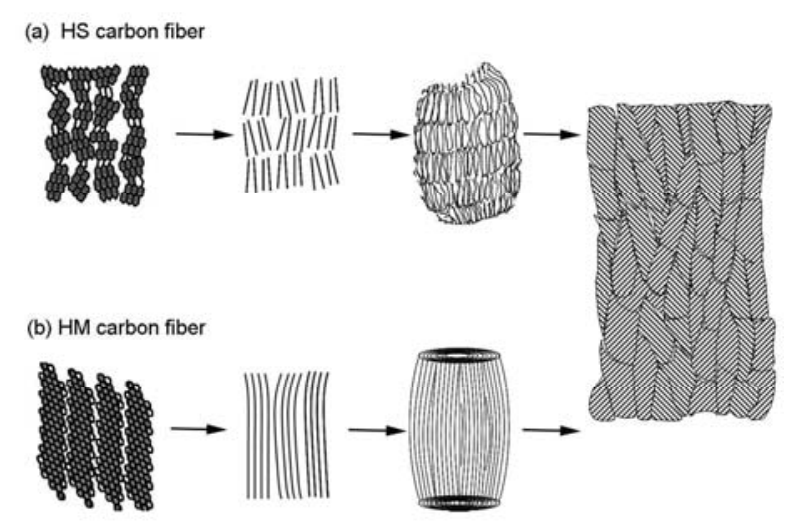

Figure 8 Structural models of PAN-based high strength and high modulus carbon fibers. Both HS and HM type's carbon fibers are composed of aggregated structure units, while the graphitic clusters within structure units are different.

A substantial fundament of this model is the mesodomain structure, however, how about the origin and development of the mesodomain in PAN-based carbon fibers? Before the discussion of this question, we would like to review the polymerization and spinning of PAN precursors. ${ }^{2}$ In the standard preparation 
process, acrylonitrile and its comonomers were polymerized in solution employing free radical initiation. The obtained copolymers with the molecular weight of 90000-140000 g/mol were further diluted in a suitable solvent such as dimethylsulfoxide to form a dope with the concentration of $15-20 \%$. The dope is pumped through a spinneret with thousands of holes into a spin bath containing normally of dilute polymer solvent. The extruded PAN copolymer coagulates in the spin bath, giving a three-dimension gel network. Herein, it should underline that the gel formation during coagulation. At the beginning of coagulation, the initial equilibrium of copolymer solution was disturbed by temperature or solvent effect, entered into a supersaturated or metastable state. Consequently, the solution becomes thermodynamically unstable and phase separation takes place via spinodal decomposition, which yields polymer-rich and solvent-rich phases. The concentrated polymer-rich phase solidifies quickly after phase separation and builds up the matrix, whereas the solvent-rich phases form the pores. Therefore, we could conclude that the polymer domain in the gel state is the origin of the mesodomain of carbon fiber, which is produced by the spinodal decomposition. After washing, plasticization drawing and drying, the solvent was removed and the pores were completely collapsed. The elongated mesodomain was shaped and stabilized due to the stretching effect. Finally, the fused mesodomain was obtained through thermal stabilization, followed by carbonization and graphitization. Therefore, the initial shape and size of mesodomains was determined by the precursor composition and spinning conditions and also strongly depended on the heat-treated temperature and stretching conditions. There is no obvious difference in the shape and size of mesodomain between the HS and HM carbon fibers, just the graphitic clusters within the mesodomain grow and coalesce to form perfect graphitic layers during graphitization.

Based on this model we predict that one kind of void could be formed among the mesodomains in the fiber texture. These voids were originated from solvent-rich phases in the coagulated fiber, and predetermined by the structure of copolymer and the conditions of its transverse and longitudinal shrinkage during plasticization drawing and drying of the fiber. Also the successive heat treatments in conjunction with the stretching strongly affect the final shape and size of voids. 
It is worth pointing out that this mesodomain model cannot give an intuitive impression of the skincore structure of fibers. The formation of skin-core structure is generally considered to the inhomogeneous oxidation of fibers as a result of the oxygen diffusion limit in the radial direction. ${ }^{25}$ Therefore, only difference in oxidation degree within mesodomain was expected instead of the change of shape and size.

\subsection{The relationship between shape and size of mesodomain and the properties}

From Figure 5, it can be concluded that the shape and size of mesodomain were determined by the structure of copolymer, and affected strongly by the process conditions. High co-polymer content could decrease the dipole-dipole repulsion between the nitrile groups, lower the spatial hindrance, and then improve the compatibility with the solvent, resulting in the large polymer domain obtained in the gel fiber. Elongation and carbonization cannot change the initial cluster size, but induce the deformation of mesodomain. High elongation, in particular, gives an obviously slender mesodomain structure. And more importantly, the tensile strength of fibers seems to relate with the size and shape of mesodomain. The smaller mesodomain, the higher tensile strength obtained for fibers. This can be explained as the more close aggregation and connection of mesodomains.

The arrangement of mesodomain is also important to determine the mechanical properties of carbon fibers. Compared mesophase pitch-based carbon fiber with the PAN-based carbon fiber, we found that the former had more ordered linear arrangement of mesodomains while the later had irregularly spiral arrangement. The spiral should have the tight junction of mesodomains. That may be one basic reason why the PAN-based carbon fibers have higher tensile strength and elongation. More comprehensive research along this line should be done in the future work.

\section{Conclusion}

By systematic examination on the structure of commercially available PAN-based carbon fibers using SEM and STM, significant mesostructure information has been obtained. High magnification SEM images revealed that both HS and HM carbon fibers had an obviously granular structure with a size of 20-40 nm on the cross section. The STM images displayed that all fibers exhibited the twisted particles 
on the longitudinal surface. The size and shape of these particles strongly depended on the precursor composition, process condition as well as the heat-treated temperature. The smaller particle, the high tensile strength obtained for the carbon fibers. According to these observations, a new model of PANbased carbon fibers, based on the mesodomain as the structural unit, was proposed. There was just a slight difference in the shape and size of mesodomain between the HS and HM carbon fibers as a result of graphitization. Further work should reveal how the size, shape and distribution of such mesodomain influence the mechanical properties of the resultant carbon fiber. The structural analysis in the present paper provide not only some direct structural evidence but also some implications for optimizing shape and size of mesodomain to achieve high-performance PAN-based carbon fibers.

\section{Reference}

1. Bahl, O.P.; Shen, Z.; Lavin J.G.; Ross, R.A. (1998) In Manufacture of carbon fibers, Donnet, J.B. (ed), New York: Marcel Dekker, 1

2. Morgan P. (2005) Carbon fibers and their composites. CRC Press Taylor \& Francis Group

3. Diefendorf, R.J.; Tokarsky, E. (1975) Polym En. Sci 15: 150

4. Ruland, W. (1990) Adv Mater 2: 528

5. Dresselhaus, M.S.; Dresselhaus, G.; Sugihara, K.; Spain, I.L.; Goldberg, H.A. (1988) In Graphite fibers and filaments. Springer Ser Mater Sci, vol.5. Berlin, Springer

6. Traceski, T.F. (1999) In Assessing industrial capabilities for carbon fiber, Acquisition and Technology, USA

7. Clarke A.J.; Bailey J.E. (1973) Nature 243: 146

8. Mochida, I.; Yoon, S.H.; Takano, N.; Fortin, F.; Korai Y.; Yokogawa, K. (1996) Carbon 34: 941

9. Goodhew, P. J.; Clarke A. J.; Bailey, J. E. (1975) Mater Sc. Eng 17: 3 
10. Fitzer, E. (1989) Carbon 27: 621

11. Bashir, Z. (1991) Carbon 29: 1081

12. Rahaman, M.S.A.; Ismail A.F.; Mustafa, A. (2007) Polym Degrad Stabi 92: 1421

13. Mittal, J.; Mathur R. B.; Bahl, O. P. (1997) Carbon 35: 1196

14. Edie, D.D. (1998) Carbon 36: 345

15. Wang, X.Z.; Jie L.; Gang, W. (2003) Carbon 41: 2805

16. Bahl, O.P.; Mathur R.B.; Kundra, K.D. (1981) Fiber Sc. Technol 15: 147

17. Fitzer, E.; Frohs W.; Heine, M. (1986) Carbon 24: 387

18. Sen, K.; Bahrami, S.H.; Bajaj, P. (1996) J Macromol Sci Rev Macromol Chem Phy C36: 1

19. Jain, M.K.; Abhiraman; A.S. (1987) J Mater Sci 22: 278

20. Jain, M.K.; Balasubramanian, M.; Desai P.; Abhiraman, A. S. (1987) J Mate. Sci 22: 301

21. Liu X.D.; Ruland, W. (1993) Macromolecules 26: 3030

22. Thiinemann, A.F.; Ruland, W. (2000) Macromolecules 33: 1848

23. Johnson, D.J.; Tyson, C.N. (1969) J Phys D Appl Phys 2: 787

24. Mencik, Z.; Plummer H.K.; Bartosiewicz, L. (1975) Carbon 13: 417

25. Bennett, S.C.; Johnson, D.J. (1979) Carbon 17: 25

26. Deurbergue, A.; Oberlin, A. (1992) Carbon 30: 981

27. Neffe, S. (1987) Carbon 25: 761

28. Barnet, F.R; Norr, M.K. (1976) Composites 7: 93 
29. Guigon, M.; Oberlin, A.; Desarmot, G. (1984) Fibr Sci Tech 20: 55

30. Guigon, M.; Oberlin, A.; Desarmot, G. (1984) Fibr Sci Tech 20: 177

31. Vezie, D.L.; Adams, W.W. (1990) J Mater Sci Lett 9: 883

32. Yoon, S.H.; Korai, Y.; Mochida, I.; Yokogawa, K.; Fukuyama, S.; Yoshimura, M. (1996) Carbon 34: 83

33. Hong, S.H.; Korai, Y.; Mochida, I. (1999) Carbon 37:917

34. Hong, S.H.; Yoon, S. H.; Mochida, I. (2000) Carbon 38:805

35. Bai, Y.J.; Wang, C.G.; Lun, N.; Wang, Y.Y.; Yu, M.J.; Zhu, B. (2006) Carbon 44: 1773

36. Hoffman, W.P. (1992) Carbon 30: 315

37. Brown, N. M. D.; You, H. X. (1990) Surf Sci 237: 273

38. Donnet, J.B.; Qin, R.Y. (1993) Carbon 31: 7 